\title{
Real-Time Monitoring System Based on Li-Fi Network Technology in Healthcare
}

\author{
Yasser Nozad Mohammedtawfiq ${ }^{1 *}$, Ayad Nozad Mohammedtawfeeq ${ }^{2}$ \\ ${ }^{1 *}$ Shaqlawa technical college, Erbil polytechnic university, Erbil, Iraq \\ ${ }^{2}$ Abo Alhail Al jaberi School, Directorate General of Education in Dhi-Qar, Dhi-Qar, Iraq \\ E-mail: ${ }^{1 *}$ Yasrnawzad@epu.edu.iq, ${ }^{2}$ Ayadnawzad84@yahoo.com
}

(Received MAy 19, 2021; Accepted August 02, 2021; Available online September 01, 2021)

DOI: 10.33899/edusj.2021.130106.1159, () 2021, College of Education for Pure Science, University of Mosul.

This is an open access article under the CC BY 4.0 license (http://creativecommons.org/licenses/by/4.0/).

\begin{abstract}
:
Patients at healthcare facilities require a long-term continuous healthcare monitoring system to keep track of their vital signs. As it deals with human life, this system must be safe, trustworthy, and does not interfere with available radio frequencies or sensitive electronic devices such as MRI (magnetic resonance imaging). This paper introduces a patient monitoring system in intensive care that uses $\mathrm{Li}-\mathrm{Fi}$ technology, designed to help enhance patient care and boost doctor's clinical results. This robust approach collects patient data timely and integrates securely within the hospital IT framework, feeding information to physicians, allowing them to make informed clinical decisions. The system uses real-time software which displays the data from different locations for assessment. It was successfully tested in the laboratory. Some measurements are discussed, which compare the received pulses to the module's line of sight (LOS) output channel to correlate the transmitted channels. In this work, experimental analysis and measurements are performed to check the efficiency of the proposed concept.
\end{abstract}

Keywords: Li-Fi Technology, E-health shield kit, VLC

$$
\begin{aligned}
& \text { تقتية شبكة Li-Fi القائمة على نظام المراقبة في الوقت الحقيقي في الرعاية الصحية } \\
& \text { ياسر نوزاد محمد توفيق1* ، اياد نوزاد محمد توفيق2 }
\end{aligned}
$$

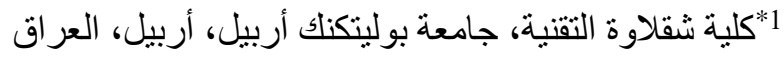

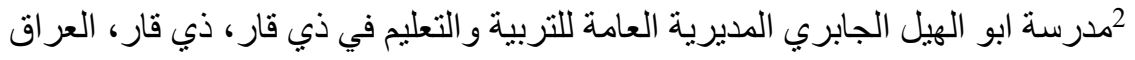

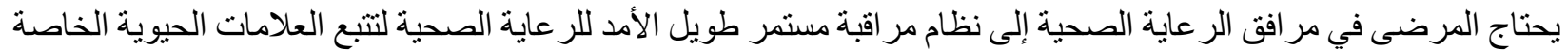

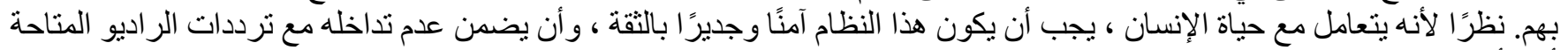

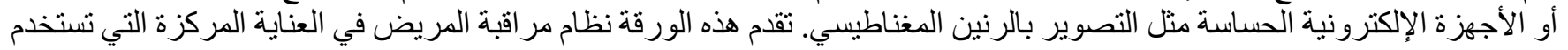

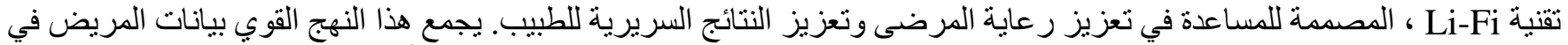

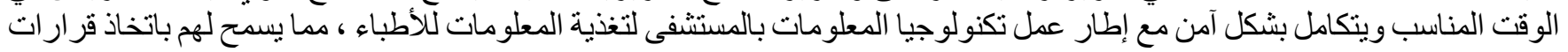

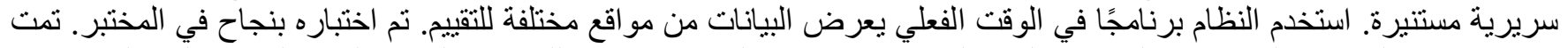

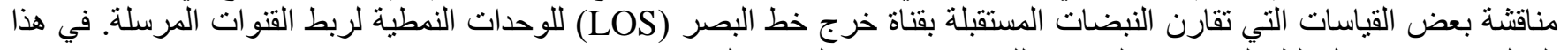
العمل ، يتم إجر اء التحليل التجريبي والقياري النياسات للتحقق من كفاءة المفهوم المقترح. 
لالكلمات المفتاحية: تقنية Li-Fi ، مجمو عة درع الصحة الإكترونية، VLC

\section{Introduction}

German physicist Harold Haas proposed Li-Fi (Light-Fidelity) as the basic solution for high-speed data networks [1]. Li-Fi networks, also known as visible light communications, enable data to be transmitted through the illumination of a light-emitting diode (LED) bulb (VLC). In the age of the Internet, there is a constant demand for quicker, safer, and more efficient wireless-wire communication in all areas, though wireless networks are favored in all domestic applications in general, and healthcare applications in particular [2].

The reason for using a wireless network in a hospital is that cables linking devices and travel over the patient's body can cause contamination. The increasing demand for radio spectrum and bandwidth places the increasing reliance on wireless internet and a burden on wireless Wi-Fi technology [3].

To relieve the strain on $\mathrm{Wi}-\mathrm{Fi}, \mathrm{Li}-\mathrm{Fi}$ is a new type of wireless internet that has applications in almost every field [4]. This system can employ many forms of modulation methods, One approach for encoding digital data through multiple carrier frequencies is orthogonal frequency division multiplexing (OFDM) [5]. The simplest type of amplitude-shift key (ASK) modulation is on-off keying (OOK), which the presence or absence of a carrier wave is used to reflect digital data [6]. Pulse-width modulation (PWM) is a modulation method that converts a message into a pulsing signal. It can be used to encode data for transmission. PPM (pulse-position modulation) is a signal modulation technique that encodes ' $\mathrm{M}$ ' message bits by transmitting a single pulse in one of several possible time-shifts that are repeated every ' $\mathrm{T}$ ' seconds [7].

This paper aims to establish? a human body healthcare continuous monitoring system based on Li-Fi technology as a safe environment to activate $\mathrm{Wi}-\mathrm{Fi}$ in hospitals to avoid electromagnetic interference. Therefore, Li-Fi offers a high-speed technology for using light as a means of transmitting data and thus providing a safer and greener environment.

As compared to $\mathrm{Li}-\mathrm{Fi}$, Wi-Fi patient monitoring is slower and also has less bandwidth. $\mathrm{Li}-\mathrm{Fi}$ has a higher level of reliability via Wi-Fi. Since data is transmitted on Wi-Fi through radiofrequency waves, these waves have the potential to affect the human body [8].

The Li-Fi technology has a $3 \mathrm{~m}$ range and allows for safe communication. VLC is the wireless transmission of information using light.

\section{Architecture System}

Figure 1. shows the monitoring framework, as a functional block diagram, whereby the basic components of the system are E-health sensors, communication channel, receiver, and transmitter. Li-Fi-based wireless healthcare monitoring system offers real-time online information about the health status of a patient by alerting health care professionals. To accomplish this, a microcontroller and a transceiver are required. The circuit diagram consists of an Arduino Uno microcontroller as a central processing unit. To input data to the microcontroller an E-health shield is needed?, the V2.0 interfacing system facilitates connection to four sensor nodes. All these sensor nodes bind the four different types of sensors, which continually sense pulse, oxygen in the blood, body temperature, ECG, airflow, of the human body, all sensor readings are collected and regulated by Arduino that sends this information to the monitoring side for processing [9]. The data obtained by the 


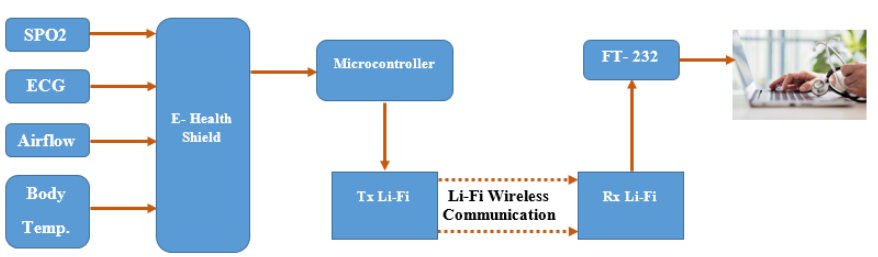

Figure 1. system architecture

microcontroller in Figure 2 ss encoded serially and transmitted using the revolutionary Li-Fi or "Light Fidelity" technology, using the light as a medium for the receiver. This system has the following topology:

Figure 3 displays the data transmission component (Tx). This uses an LED to transmit short-range wireless data. The receiving side $(\mathrm{Rx})$ Figure 4 receives the serially and optically transmitted data at a 38400 baud rate, within an effective range of 1-10 feet. Furthermore, at the transmitter side, the data is first converted to binary via an (ADC) and then fed into an LED driver circuit, which is operated by a signal processor and then modulated using on-off keying by the LED driver.
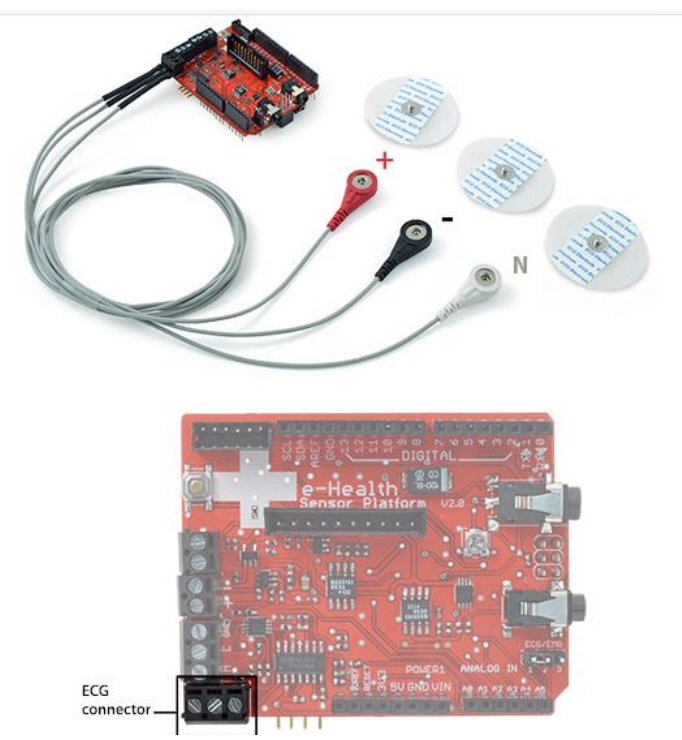

Figure 2. E-health shield

Moreover, the high-intensity LED blinks rapidly, transmitting data and optical pulses across the wireless channel. This optical transmission produces no electromagnetic interference that can influence human health or induce noise to the sensor measurements [10].

The transmitter opposed to Wi-Fi is extremely secure. The high data density of the visible light transmission can be well contained and consumes very little electricity.

These optical pulses are converted into an electrical signal by a photodetector on the receiver side, amplified by a transcendence amplifier, and then transformed back to binary data by a comparator. Then through networking the receiver to a wired Ethernet link several health care professionals can remotely access the data from different patients. 


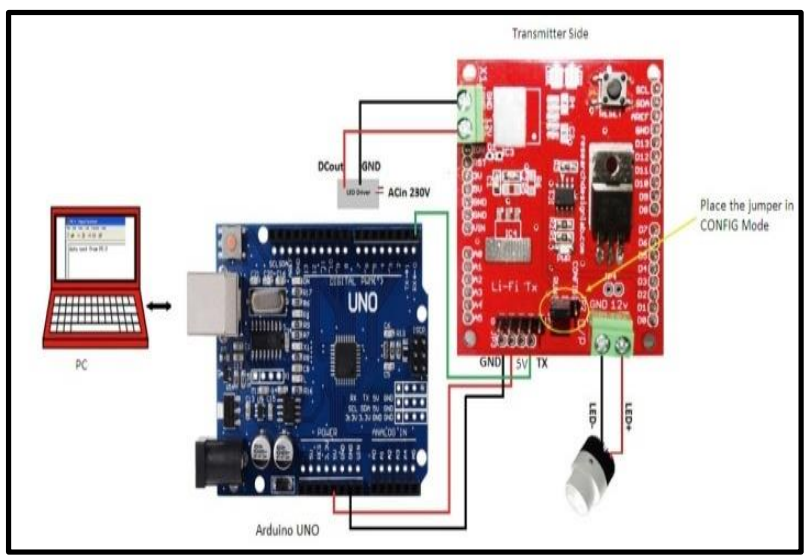

Figure 3. Tx side

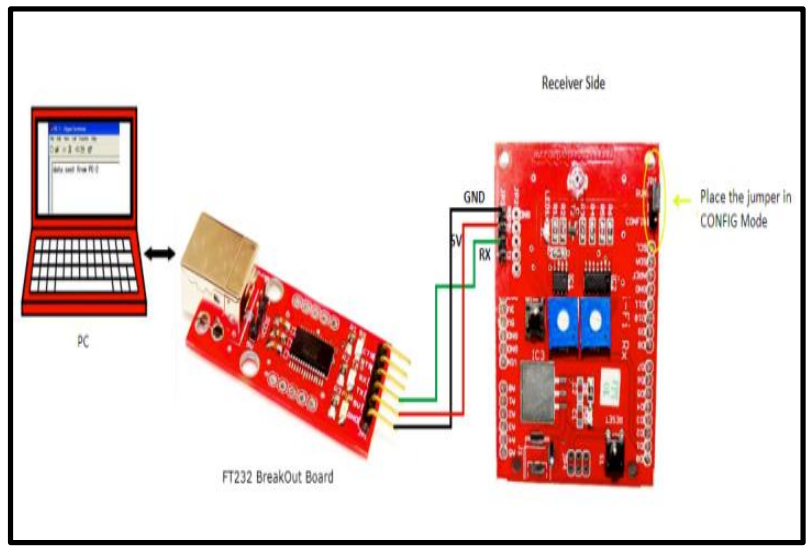

Figure 4. Rx side

\section{3- LED LIGHT DESIGN}

The luminous power and the transmitted optical force are the two fundamental properties of LED lamps. The luminous strength of LED is determined by using a system of type (Digital lux meta), the transmitted optical power indicates the total energy radiated from a LED.

To calculate the angle of illumination according to the following equations, assuming that the patient in a room 4 meters wide by 5 meters long:

$\emptyset=\cos ^{-1}\left(\frac{d^{2}}{d \sqrt{x^{2}+y^{2}+d^{2}}}\right)$

eq. (1)

Where $\phi$ is the angle formed by the light source's horizontal and the straight beam of light, $d$ is the distance between transmitter and receiver, $\mathrm{x}$ is the length of the space, $\mathrm{y}$ is the width of the room, and $\mathrm{d}$ is the horizontal distance between the led and the receiver's plan.

$\emptyset=\cos ^{-1}\left(\frac{1}{1 \sqrt{4^{2}+5^{2}+1}}\right)$

$\emptyset=81^{\circ}$

The following equation can be used to measure the direct distance between the transmitter and the receiver: 
$D_{d}=\frac{\sqrt{x^{2}+y^{2}}}{\sin \emptyset}$

$D_{d}=\frac{\sqrt{4^{2}+5^{2}}}{\sin 81}$

$D_{d}=6.48 \mathrm{~m}$

The efficiency of the device and the extent of the effect signal to interference noise ratio can be measured according to the following:

$\operatorname{SIN}=\frac{\operatorname{Ehor}(x, y, z)_{-} A C}{\operatorname{Ehor}(x, y, z)_{-} \text {Total }}$

$m=-\ln 2 / \ln \cos \phi$

Where $m$ is the order of Lambertian emission

$m=0.37$

$$
\text { Ehor }=I(0) \cos ^{m}(\phi) / D^{2} \cos (\psi)
$$

Where $I(0)$ is the center luminous intensity of an LED, is the angle of irradiance and $\psi$ is the angle of inciфence.

Digital lux meta was used to a measured LED intensity which is 3500 lx.

Ehor $=3500 * \sin ^{0.37} \frac{81}{(6.48)^{2}} * \cos (41)=11.06$

Io_depth $=I o * 0.5$

$=3500 * 0.5=1750$

$\operatorname{Ehor}(x, y, z) \_A C=\frac{I o_{\text {depth }} * \cos \left(\frac{p i}{3}\right)}{D_{d^{2}} * \cos \left(\frac{p i}{6}\right)}$

$\operatorname{Ehor}(x, y, z) \_A C=27.05$

$S I R=0.73$

\section{Results Obtained}

After completing the required calibration measures, the system was successfully activated and the data was sent and received at the speed of $(4.68 \mathrm{Mbps})$. The required samples were taken from a group of patients and saved for purposes of comparison. 

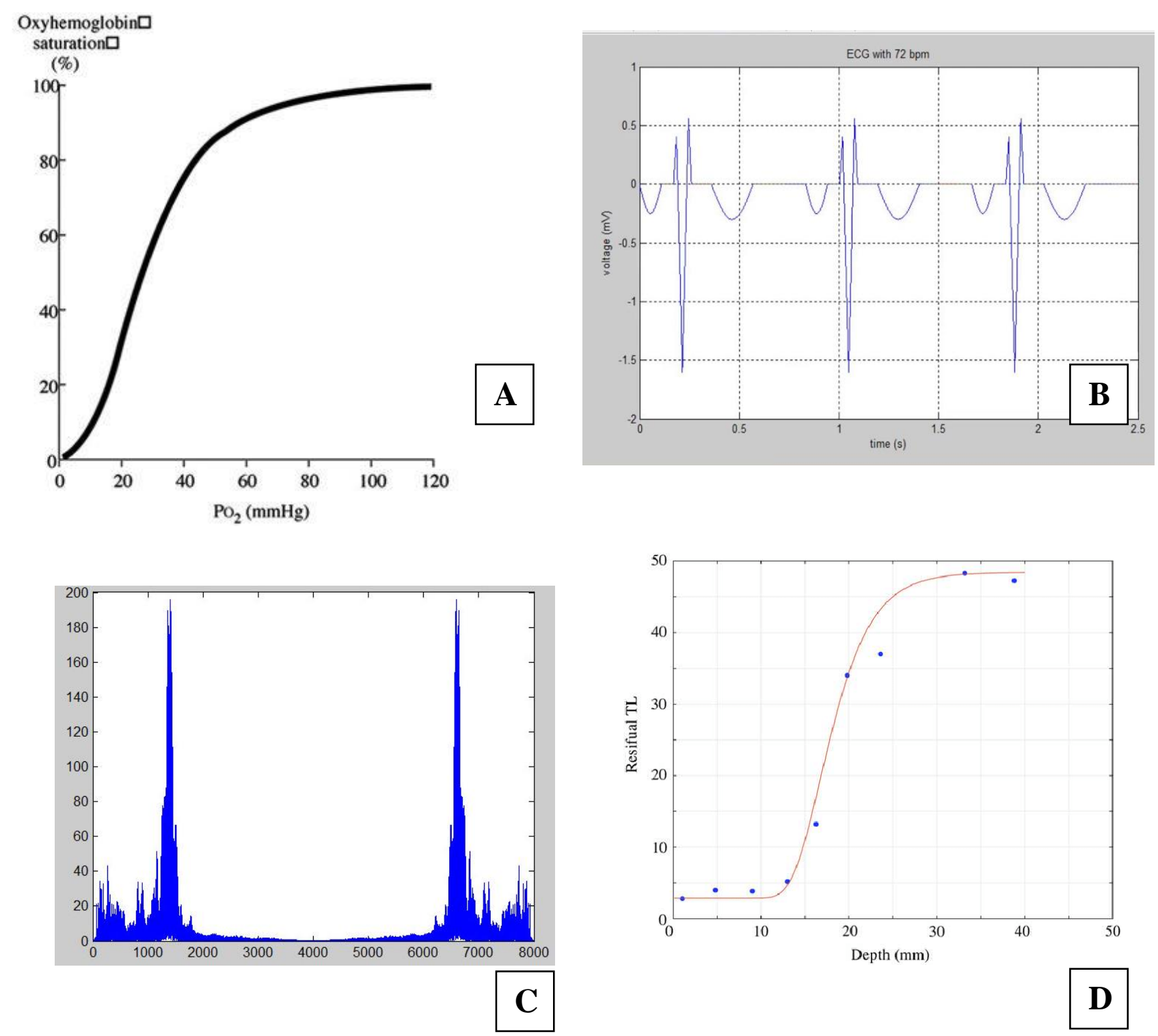

Figure 5. Data plotting for patient in real time

Figure (5-A) demonstrates the use of pulse oximetry as a non-invasive technique of determining the oxygen saturation of functional hemoglobin in the blood. In any case, where a patient's oxygenation is impaired, such as intensive care, a pulse oximeter sensor is useful, surgery, or recovery, emergency, and hospital ward settings, for assessing a patient's oxygenation and deciding whether or not supplemental oxygen is required. Patients should expect values of 95 to 99 percent, whereas those with a hypoxic drive issue should expect values of 88 to 94 percent, and values of 100 percent can indicate carbon monoxide poisoning.

The electrocardiogram (ECG) is considered one of the important problems in the medical field (hospitals and clinics), which is used to represent the health of a person, Figure (5-B) demonstrates the electrocardiogram (ECG or EKG) as a diagnostic instrument for determining the heart's muscular and electrical functions. In modern medicine, the ECG sensor has been one of the most widely used diagnostic tools. It can be used to diagnose several cardiac conditions, including Ischemia and infarction of the 
myocardium, as well as palpitations and syncope. When the patient is lying flat on a bed or table ECG leads are devoted to the body. .

Abnormalities and shifts in respiratory rate is a broad predictor of severe physiological instability. The respiratory rate is one of the first indicators of this instability. As a consequence, it is crucial to keep an eye on the respiratory rate as a sign of how the patient is doing. An AirFlow sensor, as shown in Figure (5-C), may provide early warning of hypoxemia and apnea.

Figure (5-D) indicates that the individual's body temperature is affected by the place in which the measurement is taken, as well as the time of day and level of activity. The temperature of various parts of the body varies. The average core body temperature (measured internally) is 37.0 degrees Celsius (98.6 degrees Fahrenheit). During the day, the body temperature of healthy adults fluctuate when the body's needs and behaviorsdiffer, by around $0.5^{\circ} \mathrm{C}\left(0.9^{\circ} \mathrm{F}\right)$, with lower temperatures in the morning and higher temperatures in the late afternoon and evening. Body temperature monitoring is extremely important in medicine. The reason for this is that several illnesses are accompanied by distinct changes in body temperature. Similarly, body temperature can be monitored to regulate the progression of certain diseases, and the effectiveness of remediation initiated can be calculated by the physician.

\section{Conclusion}

This paper introduces a real-time patient monitoring system using a short-range wireless connection using $\mathrm{Li}-\mathrm{Fi}$ technology. This wireless communication technology is useful because it causes no interference to sensor measurements such as ECG and allows the free movement of the patient and the medical practitioners to treat the patient. The device implementation uses very cheap components and license-free software, so it has the potential to be an incredibly cost-effective solution. The new system would help doctors who operate in busy, high-pressure settings, care for patients more efficiently and effectively. This method offers a realistic approach to using four different kinds of sensors installed on a single system. At present, four vital signs are calculated to determine the early warning score: pulse, oxygen in the blood, airflow, body temperature, and ECG. The system has been built with the future in mind so that it can easily be expanded to include other data.

\section{Aacknowledgments}

I thank Shaqlawa College of Technology for their help in cooling the scientific laboratories to accomplish this research. 


\section{References}

[1] Sarkar, A., Agarwal, S., \& Nath, A. (2015). Li-Fi technology: data transmission through visible light. International Journal of Advance Research in Computer Science and Management Studies, 3(6).

[2] Kulkarni, S., Darekar, A., \& Joshi, P. (2016, March). A survey on Li-Fi technology. In 2016 International Conference on Wireless Communications, Signal Processing and Networking (WiSPNET) (pp. 1624-1625). IEEE.

[3] Sarkar, A., Agarwal, S., \& Nath, A. (2015). Li-Fi technology: data transmission through visible light. International Journal of Advance Research in Computer Science and Management Studies, 3(6).

[4] Bhateley, P., Mohindra, R., \& Balaji, S. (2016, April). Smart vehicular communication system using Li Fi technology. In 2016 International Conference on Computation of Power, Energy Information and Commuincation (ICCPEIC) (pp. 222-226). IEEE.

[5] N. Anbu Selvan, N., Abirami, E., Arul Sangeetha, A., Beaulah Fanny, F., \& Gomathi, V. (2017). Application of LI-FI Technology in the Transmission of Sound at the Base of PWM. The SIJ Transactions on Computer Networks \& Communication Engineering (CNCE), 5 (1), 1, 4.

[6] V.P. Krishnammal., et. al., "Optimization of Visible Light Communication (VLC)". Volume 5, Special Issue 1, March 2016.

[7] Wang, Y., \& Li, H. (2013). The research of PPM modulation in space laser communication transmitting system. Sensors \& Transducers, 155(8), 195.

[8] Prakash, R., \& Agarwal, P. (2014). The new era of transmission and communication technology: Lifi (light fidelity) led \& ted based approach. International Journal of Advanced Research in Computer Engineering \& Technology (IJARCET), 3(2), 285-290.

[9] Faris, Z. M., \& Croock, M. S. (2018). Sensor Network Based Animals Feeding and Milk Production Management System. Engineering and Technology Journal, 36(2A).

[10] Karthika, R., \& Balakrishnan, S. (2015). Wireless communication using Li-Fi technology. SSRG International Journal of Electronics and Communication Engineering (SSRG-IJECE), 2(3), 32-40.

[11] Mohamed Amir Aly., et., al., "VISIBLE LIGHT COMMUNICATION SYSTEMS OVER FPGA". MSc. thesis, college of engineering, Cairo University July 2015. 